\title{
Entamoeba coli and heart diseases
}

\begin{abstract}
Entamoeba coli is a neglected intestinal amoeba, mostly occur in the tropical African countries. This species of amoeba transmit through feco-oral route, through ingestion of cyst form, which is responsible for infection and then it transforms into trophozoite stage which is pathogenic form. Most of scientists regard Entamoeba coli as a commensal intestinal amoeba, although of their bad impact on human health , it may lead to diarrhea, constipation, abdominal pain, fatigue and may lead to serious complications such as heart diseases due to electrolytes imbalance and accumulations of cholesterol and low density lipoprotein also they affect the bleeding profile. Additional studies must be conducted to reclassify this amoeba from commensal into pathogenic intestinal amoeba.
\end{abstract}

Volume 12 Issue 3 - 2019

\author{
Mosab Nouraldein Mohammed Hamad \\ Department of Medical Parasitology, Alneelain University, Sudan
}

Correspondence: Mosab Nouraldein Mohammed Hamad, Department of Medical Parasitology, FMLS, Alneelain University, Sudan, Email musab.noor13@gmail.com

Received: April 27, 2019 | Published: May 17, 2019

Keywords: amoeba, Entamoeba, coli, heart, diseases, amebic dysentery, intestinal, lipoprotein, hartmanni

\section{Opinion}

The term "amoeba" refers to uncomplicated eukaryotic organisms that move in a characteristic crawling fashion. On the other hand, a comparison of the genetic content of the various amoebae shows that these organisms are not necessarily closely related. ${ }^{1}$ The well-known type species, Amoeba proteus, is found on decaying bottom vegetation of freshwater streams and ponds. There are several parasitic amoebas. Of six species found in the human alimentary tract, Entamoeba histolytica causes amebic dysentery. Two related free-living genera of increasing biomedical importance are Acanthamoeba and Naegleria, strains of which have been recognized as disease-causing parasites in several vertebrates, including humans. ${ }^{2}$

Other species of amoeba such as Entamoeba coli, Entamoeba polecki, Entamoeba hartmanni and Iodamoeba buetschlli .These organisms have the same cycle of development, featured by a vegetative form, which lives in the large intestine, and a cyst stage, which is the infective form. These species of intestinal amoeba are differentiated from one another by their morphology. ${ }^{3}$ Entamoeba coli are regarded up to date as a protozoan endocommensel, residing the lumen of the large intestine of man. There is no reliable evidence that it produces disease in human beings but few workers have reported ingestion of red blood cells by the organism. E. coli was discovered in India by Lewis in 1870 however its detail description was given by Grassi (1879). Amoeba coli infection because high levels of cholesterol and low density lipoprotein. ${ }^{4}$ Entamoeba coli tell now considered as commensal amoeba despite their potent phagocytic role that enable it to engulf other organisms even other intestinal parasites such as Giardia lamblia whom compete it in nutrients and they already perform many useful activities inside gastrointestinal tract and the disturbance of this homeostasis may lead to serious complications one of which is cardiovascular disorders. The pathology of Entamoeba coli may be attributed to their strong phagocytic activity, which lead to reduction of gut beneficial flora, then the vital roles of that types of bacteria are disturbed .Enzymes produced by intestinal bacteria are important in the metabolism of several vitamins. The intestinal microflora synthesizes vitamin $\mathrm{K}$, which is a necessary cofactor in the production of prothrombin and other blood clotting factors. Treatment with antibiotics, particularly in individuals eating a diet low in vitamin $\mathrm{K}$, can result in low plasma prothrombin levels and a tendency to bleed. Intestinal bacteria also synthesize biotin, vitamin B12, folic acid, and thiamine. Due to their bad effect on bacteria flora it may affect the bleeding profile, further more it leads to accumulation of cholesterol and low density lipoprotein also it affects the level of phosphorus. ${ }^{5}$ For the above mentioned consequences of moderate or heavy infection with Entamoeba coli, I suggest that this species of amoeba is one of the major causes of heart diseases around the globe. Extensive studies about Entamoeba coli must be done in order to know more details about the impact of infection with type of amoeba on the man health. I am very interested to be part of any research project about the role of Entamoeba coli infection in heart diseases. Ignorance of infections which is not common in the developed countries is a big crime exits in the world despite the efforts done by world health organization to save the life of poor people around the world, but its limited efforts and the international community must raise their sense of humanity toward those people whom live in dark part of the earth.

\section{Acknowledgments}

None.

\section{Conflicts of interest}

Authors declare that there is no conflict of interest.

\section{References}

1. https://www.livescience.com/54281-amoeba-definition.html.

2. https://www.britannica.com/science/amoeba-order

3. https://www.sciencedirect.com/topics/medicine-and-dentistry/ entamoeba-coli

4. Mosab Nouraldein Mohammed Hamad, Ammar Yasir Mohamed Elhassan. Effect of Entamoeba coli on levels of cholesterol and low density lipoprotein in human plasma. Ejbps. 2017;4(9):165-167.

5. Mosab Nouraldein Mohammed Hamad, Madiha Elfadil Elkhairi, Tarig Mohamed Elfaki. Entamoeba coli infection as a risk factor for cardiovascular diseases. Ejbps. 2017;4(10):56-59. 